\title{
Hybrid Filtration Combustion
}

\author{
Mario Toledo Torres and Carlos Rosales Huerta
}

Additional information is available at the end of the chapter

http://dx.doi.org/10.5772/50353

\section{Introduction}

Recent stringent emission regulations and depletion of energy sources have imposed special requirements on combustion technologies for the new millennium. By now it is well known that hydrocarbon sources, the bedrock of economic wealth and combustion science, are being depleted 100000 times faster than they are being replenished. These factors will no doubt demand the development of novel combustion techniques. Among other concepts, combustion in a porous media offers a possible technological breakthrough and solutions for the near and long term. It can provide the basis for development for new combustion systems. The study of porous media phenomena itself can be a multidisciplinary field ranging from mechanical and chemical to geological and petroleum applications [1].

Porous media combustion, also known as filtration combustion, is defined as the process in which a self-sustaining exothermal reactive wave propagates over a porous reagent by means of gaseous oxidizer filtration through an inert solid matrix towards the reaction zone. As an internally self-organized process of heat recuperation, filtration combustion of gaseous mixtures in porous media differs significantly from the homogeneous flames. This difference is attributed to the following main factors: the highly developed inner surface of the porous medium results in efficient heat transfer between gas and solid; dispersion of the gas flowing through a porous media increases effective diffusion and heat transfer in the gas phase. To further elaborate, once a gas mixture is ignited inside the media, the heat release from the intense reaction zone is transferred to the solid matrix that subsequently feeds a fraction of the energy to the solid layers immediately above and below. This process facilitates a combustion process that ensures stability in a wide range of gas filtration velocities, equivalence ratios, and power loads.

Stationary and transient systems are the two major design approaches commonly employed in porous combustion [2-8]. The first approach is widely used in radiant burners and surface combustor-heaters where the combustion zone is stabilized within the finite element of the porous matrix. The second (transient) approach involves a traveling wave representing an 
unsteady combustion zone freely propagating in either downstream or upstream direction in the inert porous media (IPM). Strong interstitial heat transfer results in a low degree of thermal non-equilibrium between gas and solid. These conditions correspond to the lowvelocity regime of filtration gas combustion, according to classification given by Babkin [9]. The relative displacement of the combustion zone results in positive or negative enthalpy fluxes between the reacting gas and the solid matrix. As a result, observed combustion temperatures can be significantly different from the adiabatic predictions and are controlled mainly by the reaction chemistry and heat transfer mechanism. The upstream wave propagation, countercurrent to filtration velocity, results in under-adiabatic combustion temperatures, while the downstream propagation of the wave leads to the combustion in a super-adiabatic regime with temperatures much in excess of the adiabatic one. Superadiabatic combustion significantly extends conventional flammability limits to the region of ultra-low heat content mixtures.

Superadiabatic filtration combustion of rich and ultra-rich mixtures creates a situation in which partial oxidation and/or thermal cracking of hydrocarbons take place. This technology for hydrogen or synthesis gas (or syngas, a gas mixture that contains varying amounts of carbon monoxide and hydrogen) production uses an IPM [10-13]. The fuels used in porous combustion systems are basically of gaseous form due to fluidity, volumetric capacity, and shorter mixing length scale [14-21]. Liquid fuel reactors have been developed and used to a smaller extent [22-30].

According with the theory and technology of filtration combustion and the required new combustion systems, a new hybrid porous reactor can be developed changing an inert solid volume fraction by solid fuels. Now the porous medium is composed of uniformly mixed aleatory solid fuel and inert particles [31]. This change produces hybrid filtration combustion for simultaneous conversion of solid and gaseous fuels to energy (lean combustion) or hydrogen and synthesis gas (rich combustion).

As a result of composed porous medium, observed combustion temperatures can be lower than the IPM values and are controlled mainly by the heterogeneous reaction chemistry and heat transfer mechanism. Upstream and downstream wave propagations are present in the hybrid porous reactor. The upstream wave propagation in the composite bed is similar to the inert bed but the downstream wave propagation show a flat temperature profile with practically constant temperature along the reactor.

The combustion temperature in composite bed decreases from rich to ultra-rich gas fuel-air mixtures, and also with the increase of solid fuel volume fraction in porous medium. That suggests a change of dominant kinetic mechanism and a shift from homogeneous to heterogeneous chemistry. At high downstream propagation velocities near the stoichiometric conditions the role of gas phase kinetics is dominant. In these conditions, the upstream wave propagation suppresses the heterogeneous oxidation processes by the complete consumption of oxidizer. At higher equivalence ratios, the wave propagation is slower. This increases the role of heterogeneous kinetics. As a result, the ignition and combustion temperatures drop. In turn, this affects the wave velocity. The mechanism of heterogeneous combustion becomes dominant for downstream wave propagation. The 
slowly reacting solid fuel is directly exposed to oxidizer. The ignition initiated over the solid phase is further transferred to the gas phase. It is possible to suggest that the structure of the wave in this case involves a heterogeneous reaction front followed by the gas phase reaction front. These fronts are followed by a slow endothermic reaction between the formed steam and solid/gaseous fuels.

The propagation of downstream hybrid combustion waves in the fuel loaded medium has resulted in wide combustion fronts with superadiabatic combustion temperatures. Due to the wide flat temperature profiles it has been found impossible to determine the wave velocity with sufficient accuracy. The flat temperature profile also suggests that the fuel particles are burning upstream of the front.

The use of rich and ultra-rich mixtures of gas fuel with air hybrid filtration combustion for hydrogen and syngas production has big potential. Hydrogen and syngas concentration increases with an increase of equivalence ratio for gas fuel/air mixtures in the inert porous medium and composite bed. In comparison, more high concentration of hydrogen and syngas is obtained in the composite porous media. Hydrogen conversion for gas fuel/air mixtures for hybrid filtration combustion waves is $50 \%$ for higher equivalence ratio.

\section{Physical and mathematical description of the hybrid process}

Lean combustion results in complete burnout of the hydrocarbon fuel, with the formation of carbon dioxide and water. Thus, both the composition of the final products and the heat release are well defined. In contrast to the lean case, the partial oxidation products of rich and ultra-rich waves are not clearly defined. In such a case, fuel is only partially oxidized in the wave, and the total heat release could be kinetically controlled by the degree of partial oxidation. As a result, chemical kinetics, heat release, and heat transfer are strongly coupled in the rich and ultra-rich waves, rendering it a more complicated and challenging phenomenon than the lean wave.

In the following we describe the governing equations, derived from fundamental principles, as well as some suitable models and assumptions that allow the formulation of a mathematical model. Such a model can be tractable by numerical simulation. We consider the bulk volume $V$ of the bed separated into the volume occupied by the solid particles, $V_{s}$, and the volume occupied by the gas $V_{g}$ (i.e. $V=V_{g}+V_{s}$ ). As usual, we define the porosity $\theta$ as the fraction of bulk volume $V$ occupied by the gas: $V_{g}=\theta V$. The composition of the gas is characterized by the molar fractions $\left\{y_{j}, j=1, \ldots, N_{S P}\right.$ of the $N_{S P}$ chemical species in the gas phase. On the other hand, the solid phase is composed of reacting fuel particles and inert particles. For the sake of conciseness, in this section we will refer to this solid fuel simply as fuel (it is implicit, by our definition of hybrid filtration combustion, that some of the species in the gas phase are also components of a gaseous fuel injected into the porous medium). To specify the composition of this solid medium we define partial (mass) densities for the fuel and inert gas as

$$
\rho_{f}=\frac{m_{f}}{V_{s}} ; \quad \rho_{i}=\frac{m_{i}}{V_{s}}
$$


where $m_{f}$ and $m_{i}$ are respectively the mass of fuel and the mass of inert component in a given volume $V$. Note that these densities are based on the solid volume, so that $m_{f}=\rho_{f} \theta V$, and similarly for $m_{i}$. Here and in all this chapter the subscripts $(g)$ refer to the gas phase, $(s)$ to the solid phase, $(f)$ to the fuel component in the solid, and (i) to the inert component in the solid.

\subsection{Conservation of mass for gaseous species}

For an arbitrary volume $V$ in the porous medium, the conservation of mass for the $j$-th species in the gaseous mixture is given by

$$
\int_{V} \frac{\partial}{\partial t}\left(\theta \rho_{g} y_{j}\right) \mathrm{d} V+\int_{A} \rho_{g} y_{j} \overrightarrow{\mathbf{u}} \cdot \overrightarrow{\mathbf{n}} \mathrm{d} A+\int_{A} \rho_{g} y_{j} \overrightarrow{\mathbf{w}}_{j} \cdot \overrightarrow{\mathbf{n}} \theta \mathrm{d} A=\int_{V} S_{j} \mathrm{~d} V .
$$

The first term on the left-hand side (LHS) is of course the local rate of variation of the molar quantity of species $j$ in the volume, where $\rho_{g}$ is the molar density of the gas. The second term is the advection of species $j$ by the gas flowing out of $V$ through is bounding surface $A$ ( $\overrightarrow{\mathbf{n}}$ is the outward normal vector). This flow is calculated using the filtration velocity $\overrightarrow{\mathbf{u}}$, so that the integration is taken over the whole area $A$. The third term is the additional transport across that bounding surface due to the diffusion velocity, $\overrightarrow{\mathbf{w}}_{j}$, of species $j$ relative to the mean gas velocity. The right-hand side contains the integration of the source term, $S_{j}$, per unit of bulk volume. This term contains the net production of species $j$ by homogeneous reaction in the gas phase, heterogeneous reaction with the solid fuel, and volatile matter release by that fuel, as explained later.

Although the diffusion velocities $\overrightarrow{\mathbf{w}}_{j}$ can in principle be obtained by solving a system of equations, involving the binary mass diffusion coefficients for each pair of species in the gas, such treatment is computationally difficult and expensive. Therefore, the usual simplified approach is based on Fick's law

$$
y_{j} \overrightarrow{\mathbf{w}}_{j}=-D_{j} \nabla y_{j}
$$

where $D_{j}$ is the diffusion coefficient of species $j$ into the gaseous mixture. Hence, from Eq. (2) one readily obtains

$$
\frac{\partial}{\partial t}\left(\theta \rho_{g} y_{j}\right)=\nabla \cdot\left(\theta \rho_{g} D_{j} \nabla y_{j}\right)-\nabla \cdot\left(\rho_{g} \overrightarrow{\mathbf{u}} y_{j}\right)+S_{j} ; \quad \text { for } j=1, \ldots, N_{S P}
$$

Among other parameters, the filtration velocity $\overrightarrow{\mathbf{u}}$ must be known in Eqs. (4) in order to solve them for $y_{j}$. In many applications with porous media in reactors of simple geometry (for instance, a long and narrow cylinder), the filtration velocity is determined by a global mass balance of the reactor, and the whole problem can be analyzed as one-dimensional.

By their definition, the mole fractions must satisfy

$$
\sum_{j=1}^{N_{S P}} y_{j}=1
$$


so that, one of the Nsp equations (4) can be eliminated, and the associated mole fraction can be obtained by difference from Eq. (5). We remark that, as shown in Ref. [32], solving (NSP 1) species equations in this way can produce numerical problems, when the exact diffusion velocities have been substituted by Fick's law, conducing to a violation in global mass conservation. The error vanishes when all diffusion coefficients are equal $\left(D_{j}=D\right)$, and it is negligible if the species for which the equation was removed has a relatively high concentration in the mixture.

The gas volume $V_{g}$ in the region $V$ receives chemical species which come from: (i) advection by the gas flowing into $V_{g}$, (ii) enter $V_{g}$ as a component of volatiles evolved from the solid fuel, or (iii) enter $V_{g}$ as a product of heterogeneous reactions of the solid fuel with gaseous species. The species will react in the gaseous phase according to a homogeneous reaction mechanism. The presence of heterogeneous reactions means that simultaneously some of these species will be consumed in such reaction. The superposition of these processes determine the net source term $S_{j}$ in the equation (4).

\subsubsection{Homogeneous reaction}

The homogenous reaction of the NSP species in the gas is generally expressed as a system of NHO reactions

$$
\sum_{j=1}^{N_{S P}} \eta_{j \alpha}^{\prime} \mathrm{M}_{j} \longleftrightarrow \sum_{j=1}^{N_{S P}} \eta_{j \alpha}^{\prime \prime} \mathrm{M}_{j} \quad ; \quad \text { for } \alpha=1, \ldots, N_{H O}
$$

where $\mathrm{M}_{j}$ represents symbolically the molecule of species $j$, and $\left(\eta_{j \alpha}^{\prime}, \eta_{j \alpha}^{\prime \prime}\right)$ are the stoichiometric coefficients of the $j$-th species in the $\alpha$-th homogeneous reaction. These reactions are considered, in principle, reversible so that the rates of consumption and production of species $j$ are given by

$$
\sum_{\alpha=1}^{N_{\text {HO }}} \eta_{j \alpha}^{\prime} r_{f \alpha}+\eta_{j \alpha}^{\prime \prime} r_{b \alpha} \text { and } \sum_{\alpha=1}^{N_{\text {HO }}} \eta_{j \alpha}^{\prime \prime} r_{f \alpha}+\eta_{j \alpha}^{\prime} r_{b \alpha}
$$

respectively. Here, $r_{f \alpha}$ is the forward reaction rate $(\rightarrow)$, and similarly $r_{b \alpha}$ is the backward reaction rate $(\leftarrow)$. Thus, the net production for the $j$-th species by the homogeneous reaction mechanism is

$$
S_{j, H O}=\sum_{\alpha=1}^{N_{H O}}\left(\eta_{j \alpha}^{\prime \prime}-\eta_{j \alpha}^{\prime}\right)\left(r_{f \alpha}-r_{b \alpha}\right)
$$

The forward and backward reaction rates can be obtained from the law of mass action. Considering the mechanism (6) as composed of elementary reactions, the order of the reactions corresponds to the stoichiometric coefficients, and therefore

$$
r_{f \alpha}=k_{f \alpha} \rho_{g}^{n_{\alpha}^{\prime}} \prod_{j=1}^{N_{S P}} y_{j}^{\eta_{j \alpha}^{\prime}} ; \text { with } n_{\alpha}^{\prime}=\sum_{j=1}^{N_{S P}} \eta_{j \alpha}^{\prime}
$$


and

$$
r_{b \alpha}=k_{b \alpha} \rho_{g}^{n_{\alpha}^{\prime \prime}} \prod_{j=1}^{N_{S P}} y_{j}^{\eta_{j \alpha}^{\prime \prime}} ; \text { with } n_{\alpha}^{\prime \prime}=\sum_{j=1}^{N_{S P}} \eta_{j \alpha}^{\prime \prime}
$$

In these expressions, $k_{f \alpha}$ and $k_{b \alpha}$ correspond to the forward and backward kinetic factors, which are usually modelled using the Arrhenius law

$$
k_{f \alpha}=A_{f \alpha} T_{g}^{b_{\alpha}} \exp \left(-\frac{E_{f \alpha}}{R T_{g}}\right)
$$

where the constants $A_{f \alpha}$ and $b_{\alpha}$, as well as the activation energy $E_{f \alpha}$, are data to be provided for each reaction. These parameters constitute an essential part of the chemical reaction model (and one of the most difficult to obtain). The backward kinetic factors are computed from the forward factors using the equilibrium constants $K_{\alpha}$ of the reactions

$$
k_{b \alpha}=\frac{k_{f \alpha}}{K_{\alpha}} \text { with } K_{\alpha}=\left(\frac{p_{o}}{R T_{g}}\right)^{n_{\alpha}^{\prime \prime}-n_{\alpha}^{\prime}} \exp \left(\sum_{j=1}^{N_{S P}}\left(\eta_{j \alpha}^{\prime}-\eta_{j \alpha}^{\prime \prime}\right) \frac{g_{j}^{o}}{R T_{g}}\right)
$$

where $p_{o}$ is the pressure of the standard state ( $p_{0}=1 \mathrm{bar}$ ), $R$ is the universal gas constant and $g_{j}$ is the Gibbs free energy of species $j\left(g_{j}=h_{j}-T s_{j}\right)$. These properties depend on gas temperature, and can be obtained from available regression functions, which can be written as polynomials in $T_{g}$ such as

$$
h_{j}=\sum_{n=0}^{m} a_{n j} T_{g}^{n}
$$

for the enthalpy, and as

$$
s_{j}=\sum_{n=0}^{m-1} b_{n j} T_{g}^{n}+b_{m} \ln T_{g}
$$

for the entropy (as, for example, NASA polynomials).

\subsubsection{Volatiles release}

The process of volatiles release from the solid fuel, by thermal decomposition and pyrolysis, can be modelled as one or several irreversible reactions, which we can represent symbolically as

$$
\begin{array}{ccc}
\{\text { Fuel }\} & \stackrel{k_{1}^{(v)}}{\longrightarrow} & \mu_{1} \mathrm{~V}_{1}+\left(1-\mu_{1}\right) \mathrm{S}_{1} \\
\{\text { Fuel }\} & \stackrel{k_{2}^{(v)}}{\longrightarrow} & \mu_{2} \mathrm{~V}_{2}+\left(1-\mu_{2}\right) \mathrm{S}_{2} \\
\vdots & \vdots & \vdots \\
\{\text { Fuel }\} & \stackrel{k_{N v}^{(v)}}{\longrightarrow} & \mu_{N_{v}} \mathrm{~V}_{N_{v}}+\left(1-\mu_{N_{v}}\right) S_{N_{v}}
\end{array}
$$


to indicate the conversion of the solid fuel into volatiles $\left\{\mathrm{V}_{1}, \mathrm{~V}_{2}, \ldots, \mathrm{V}_{\mathrm{Nv}}\right\}$, all of which have a prescribed chemical composition. The constants $\left\{\mu_{\beta}, \beta=1, \ldots, N_{v}\right\}$, which are also parameters of the model, denote the fraction of fuel mass that is transformed into the volatile $V_{\beta}$ via the $\beta$-th reaction in the system (14). Such transformation leaves a solid substance $S_{\beta}$ which will react heterogeneously with the surrounding gas (as, for example, coke when coal is being burned). For each of these $N_{v}$ reactions, the model postulates a kinetic factor that can be put into an Arrhenius form

$$
k_{\beta}^{(v)}=A_{\beta} T_{s}^{b_{\beta}} \exp \left(-\frac{T_{\beta}}{T_{s}}\right) \text { for } \beta=1, \ldots, N_{v}
$$

as a function of the solid particles temperature $T_{s}$. These factors are defined such that for a given mass of fuel $m_{f}$, the rate at which mass of volatile $\mathrm{V}_{\beta}$ is released into the gas phase is given by

$$
r_{\beta}^{(v)}=k_{\beta}^{(v)} \mu_{\beta} m_{f}
$$

The model requires the activation temperatures $T_{\beta}$, the pre-exponential constants, and the composition of the released volatiles. Typically for coal, the species present in the volatiles are $\mathrm{H}_{2}, \mathrm{H}_{2} \mathrm{O}, \mathrm{CO}, \mathrm{CO}_{2}, \mathrm{CH}_{4}$ and some other hydrocarbons. The composition can be defined in terms of the mass fraction of the species present in such gaseous mixtures. We use $\sigma_{j \beta}$ to indicate the mass fraction of species $j$ in the volatile $\beta$. Therefore, superposing the effects of the $N_{v}$ vias of volatilization, the source of species $j$ in the gas phase, per unit of bulk volume, is

$$
S_{j, v}=\frac{(1-\theta) \rho_{f}}{W_{j}} \sum_{\beta=1}^{N_{v}} k_{\beta}^{(v)} \mu_{\beta} \sigma_{j \beta}
$$

due to the volatilization process. $W_{j}$ is the molecular weight of species $j$ and $\rho_{f}$ is defined in Eq.(1).

\subsubsection{Heterogeneous reaction}

The solid substances $S_{\beta}$ left by the volatilization mechanism can further react with several species from the surrounding gas. This heterogeneous reactions can be treated in a similar way to what is done in 2.1.1 for the homogeneous reactions. First, we need to define precisely the solids $S_{\beta}$. When the solid fuel is coal, the usual assumption is to consider $S_{\beta}$ as pure carbon for all the volatilization reactions (14). Global heterogeneous reactions that can take place in such conditions are for example

$$
\begin{aligned}
& 2 \mathrm{C}+\mathrm{O}_{2} \longrightarrow 2 \mathrm{CO} \\
& \mathrm{C}+\mathrm{O}_{2} \longrightarrow \mathrm{CO}_{2} \\
& \mathrm{C}+\mathrm{CO}_{2} \longrightarrow 2 \mathrm{CO} \\
& \mathrm{C}+\mathrm{H}_{2} \mathrm{O} \longrightarrow \mathrm{CO}+\mathrm{H}_{2}
\end{aligned}
$$


For some other fuels, whose fixed carbon fraction is mostly C, such as wood pellets, the treatment of $S_{\beta}$ as $C$ could also be a good approximation. Alternatively, a more accurate representation of $\mathrm{S}_{\beta}$ could be a pseudo-molecule $\mathrm{C}_{x} \mathrm{H}_{y}$, that can be used to represent coke or other form of carbonized material. Since we want to consider the solid fuel as just one component of the solid phase, in addition to the inert material, the heterogeneous reactions cannot discriminate between different solid species, and therefore the heterogeneous reaction mechanism must be written as

$$
\mathrm{S}+\sum_{j=1}^{N_{S P}} v_{j \gamma}^{\prime} \mathrm{M}_{j} \longrightarrow \sum_{j=1}^{N_{S P}} v_{j \gamma}^{\prime \prime} \mathrm{M}_{j} \text { for } \gamma=1, \ldots, N_{H E}
$$

where $N_{H E}$ is the number of reactions in the heterogeneous mechanism and $\mathrm{S}$ denotes the molecular formulae for the solid material left by the pyrolysis ( $\mathrm{C}$ or $\left.\mathrm{C}_{x} \mathrm{H}_{y}\right)$. As before, $\mathrm{M}_{j}$ stands for the molecule of $j$-th species in the gas phase, and the constants $\left(v_{j \gamma}^{\prime}, v_{j \gamma}^{\prime \prime}\right)$ are the stoichiometric coefficients for the $j$-th species in the $\gamma$-th heterogeneous reaction. A more general treatment would be to consider different solid species $S_{\beta}$ generated by the volatilization reactions. In that case, different $S$ molecules could appear for different $\gamma$ in the equations (19). The model for the kinetics could be essentially the same than the one shown below, but such an approach would require to characterize the solid phase by the content of inert material and more than one solid fuel species: $\rho_{i}, \rho s 1, \rho s 2, \ldots$, instead of $\rho_{i}$ and $\rho f$. Consequently, that would demand to solve mass conservation equations for $\rho s 1, \rho s 2, \ldots$, instead of just one for $\rho_{f}$ (See 2.2). Although in conceptual terms there is no more complexity in such a procedure, we prefer do not follow that line here, in order to avoid the introduction of too many parameters, which are currently difficult to obtain and validate.

The mass rate of consumption, per unit of bulk volume, through the $\gamma$-th reaction of the fuel that has remained solid (in the S form), can be formulated as

$$
r_{\gamma}^{(H)}=k_{f \gamma}^{(H)} \prod_{j=1}^{N_{S P}}\left(\rho_{g} y_{j}\right)^{v_{j}^{\prime}}(1-\omega) F
$$

where $\omega$ and $F$ are defined below, and $k_{f \gamma}^{(H)}$ is a kinetic factor defined such that when multiplied by the product of the concentrations in Eq.(20), gives the rate of mass consumption per unit area of solid fuel. This kinetic energy can be postulated to follow as usual an empirical Arrhenius law

$$
k_{f \gamma}^{(H)}=A_{\gamma}^{(H)} T_{s}^{b_{\gamma}^{(H)}} \exp \left(-\frac{E_{\gamma}}{R T_{s}}\right)
$$

for which the activation energy $E_{\gamma}$ and pre-exponential constants must be provided. The last two factors in Eq. (20) takes into account the surface area of solid fuel per unit of bulk volume. $F$ is the ratio of particles surface area to the volume of porous medium. For a homogeneous porous medium with particles of characteristic mean size $d$, it has the value 


$$
F=\frac{6(1-\theta)}{d}
$$

The factor $(1-\omega)$ is the fraction of $F$ that corresponds to solid fuel area. The variable $\omega$ can be defined as the degree of fuel burnout, since $\omega=1$ corresponds to the state when all the fuel that remained in the solid phase has been consumed. The minimum value for $\omega$ depends on the initial fraction of fuel in the porous medium (see 2.2).

Taking into account the production and consumption of gaseous species by the reactions (19), the net production of species $j$ by the whole heterogeneous reactions mechanism is then

$$
S_{j, H E}=\frac{1}{W_{\mathrm{S}}} \sum_{\gamma=1}^{N_{H E}}\left(v_{j \gamma}^{\prime \prime}-v_{j \gamma}^{\prime}\right) r_{\gamma}^{(H)}
$$

where $W_{s}$ is the molecular weight of S. We have taken reactions (19) as irreversible, but if there were reversible reactions among them, the treatment can be done in the same way as in subsection 2.1.1, applying the equilibrium constants to obtain backward reaction rates that should be subtracted from $r_{\gamma}^{(H)}$ in Eq. (23).

In summary, the species mass conservation equations can be written more explicitly as

$$
\frac{\partial}{\partial t}\left(\theta \rho_{g} y_{j}\right)=\nabla \cdot\left(\theta \rho_{g} D_{j} \nabla y_{j}\right)-\nabla \cdot\left(\rho_{g} \overrightarrow{\mathbf{u}} y_{j}\right)+S_{j, H O}+S_{j, v}+S_{j, H E} ; \quad \text { for } j=1, \ldots, N_{S P}
$$

where the net sources of species $j$ by homogeneous reactions, volatilization and heterogeneous reactions are given by Eqs. (7), (17) and (23), respectively.

\subsection{Conservation of mass for solid fuel}

To derive an equation expressing the mass conservation of solid fuel we consider first a region of volume $V(t)$, in the burning porous medium, whose boundary surface moves with the local velocity $\overrightarrow{\mathbf{v}}$ at which the solid fuel combustion propagates. In that situation the mass conservation of fuel is given by

$$
\frac{\mathrm{d}}{\mathrm{d} t} \int_{V(t)} \rho_{f}(1-\theta) \mathrm{d} V=-\int_{V(t)} B_{f} \mathrm{~d} V
$$

where $B_{f}$ is the rate of mass fuel consumption, per unit volume, by heterogeneous reaction and volatilization. The derivative on the LHS of Eq. (25) gives

$$
\int_{V(t)} \frac{\partial}{\partial t}\left[\rho_{f}(1-\theta)\right] \mathrm{d} V+\int_{A(t)} \rho_{f}(1-\theta) \overrightarrow{\mathbf{v}} \cdot \overrightarrow{\mathbf{n}} \mathrm{d} A=-\int_{V(t)} B_{f} \mathrm{~d} V
$$

whence the governing equation for the partial density of fuel in the solid phase becomes 


$$
\frac{\partial}{\partial t}\left[\rho_{f}(1-\theta)\right]+\nabla \cdot\left[\rho_{f}(1-\theta) \overrightarrow{\mathbf{v}}\right]=-B_{f}
$$

The loss of mass of fuel by volatilization is given by the summation of the $S_{j, v}$ terms in Eq. (17), for all the species. On the other hand, the loss of fuel mass due to heterogeneous reactions is given by the summation of all the consumption rates in Eq. (20), for all of such reactions. Therefore,

$$
\frac{\partial}{\partial t}\left[\rho_{f}(1-\theta)\right]+\nabla \cdot\left[\rho_{f}(1-\theta) \overrightarrow{\mathbf{v}}\right]=-\sum_{j=1}^{N_{S P}} S_{j, v}-\sum_{\gamma=1}^{N_{H E}} r_{\gamma}^{(H)}
$$

The evaluation of the rates $r_{\gamma}^{(H)}$ requires the degree of fuel burnout $\omega$. Denoting by $\hat{\rho}_{i}$ and $\hat{\rho}_{f}$ the intrinsic densities (true densities) of the inert material and the fuel respectively, $\omega$ can be obtained from the relations

$$
\rho_{f}+\rho_{i}=\hat{\rho}_{f} \frac{V_{f}}{(1-\theta) V}+\hat{\rho}_{i}\left(1-\frac{V_{f}}{(1-\theta) V}\right)
$$

and

$$
(1-\omega) F=\frac{A_{f}}{V}=\frac{6}{d} \frac{V_{f}}{V}
$$

where $V_{f}$ represents the volume occupied by the fuel particles in a given bulk volume $V$, and $A_{f}$ represents their surface area. Combining Eqs. (29), (30) and (22) we have

$$
\omega=\frac{\hat{\rho}_{f}-\left(\rho_{f}+\rho_{i}\right)}{\hat{\rho}_{f}-\hat{\rho}_{i}}
$$

so that the value of $\omega$, at a given position, can be determined using the instantaneous local values of $\rho_{f}$ and $\rho_{i}$ at that position.

\subsection{Conservation of energy of the gas phase}

For the analysis of energy conservation in the gas, we consider the thermal and the chemical energies of the gaseous species, and neglect the kinetic energy of the flow. The energy per mole of gas is then $e_{g}=h_{g}-p / \rho_{g}$, with $h_{g}$ being the total enthalpy per mole of gas mixture

$$
h_{g}=\sum_{j=1}^{N_{S P}} y_{j} h_{j}
$$

The enthalpies of the different species $\left(h_{j}\right)$ include their enthalpy of formation (and they can be computed with functions like Eq. (12)). Proceeding similarly to the analysis of mass conservation, an integral balance of energy in a volume $V$ results in 


$$
\int_{V}\left[\frac{\partial}{\partial t}\left(\theta \rho_{g} e_{g}\right)+\nabla \cdot\left(\rho_{g} e_{g} \overrightarrow{\mathbf{u}}\right)\right] \mathrm{d} V=\int_{V} H_{V} \mathrm{~d} V+\int_{A} H_{A} \theta \mathrm{d} A
$$

where $H_{V}$ refers to sources of energy per unit of bulk volume, and $H_{A}$ denote energy fluxes going into the volume across the boundary surface of $V$, per unit of gas-phase area. We have also neglected here the work done on the gas by surface forces (such as pressure and viscous stresses), and body forces (such as gravity). Those mechanical effects on the total energy are clearly negligible in comparison with the thermal effects.

The fluxes in $H_{A}$ can be joined into a vector $\overrightarrow{\mathbf{q}}$ such that $H_{A}=-\overrightarrow{\mathbf{q}} \cdot \overrightarrow{\mathbf{n}}$, and then Eq. (33) leads to the differential equation

$$
\frac{\partial}{\partial t}\left(\theta \rho_{g} e_{g}\right)+\nabla \cdot\left(\rho_{g} e_{g} \overrightarrow{\mathbf{u}}\right)=H_{V}-\nabla \cdot \overrightarrow{\mathbf{q}}
$$

Generally, it is preferred to use the enthalpy as the primitive variable for the gas energy equation. In that case Eq. (34) reads as

$$
\frac{\partial}{\partial t}\left(\theta \rho_{g} h_{g}\right)+\nabla \cdot\left(\rho_{g} h_{g} \overrightarrow{\mathbf{u}}\right)=\frac{\partial(\theta p)}{\partial t}+\nabla \cdot(p \overrightarrow{\mathbf{u}})+H_{V}-\nabla \cdot \overrightarrow{\mathbf{q}}
$$

The pressure terms appearing in (35) are also associated with mechanical energy of the flow. They contribute in part to the energy of deformation due to gas expansion (which is stored in the gas as thermal energy), and also to the flow acceleration due to pressure gradients (which changes the kinetic energy). As stated above, we consider these mechanical energy contributions negligible, in comparison with the thermal energy in a reactor. Consequently, the first two terms on the RHS of Eq. (35) are discarded.

The energy flux vector $\overrightarrow{\mathbf{q}}$ contains the heat diffusion, which can be expressed by Fourier's law, and the additional transport of energy due to the diffusion of species, with different enthalpies, in the multi-component gas

$$
\overrightarrow{\mathbf{q}}=-\lambda_{g} \nabla T_{g}+\sum_{j=1}^{N_{S P}} \rho_{g} y_{j} \overrightarrow{\mathbf{w}}_{j} h_{j}=-\lambda \nabla T_{g}-\rho_{g} \sum_{j=1}^{N_{S P}} h_{j} D_{j} \nabla y_{j}
$$

In this equation we have introduced again Fick's law for the diffusion velocities, while $\lambda_{g}$ is the gas thermal conductivity.

According to the processes described previously, the energy source term $H_{V}$ must contain (i) $H v, v$ : the enthalpy carried into the gas by the volatiles evolved from the solid fuel, (ii) $H v, H E$ : the energy released toward the gas by the heterogeneous reactions of the solid fuel and (iii) $H_{v, Q}$ : the heat transfer from the solid phase (inert material and fuel) to the gas.

With regards to the enthalpy flow contributed by the volatiles, it can be calculated as

$$
H_{V, v}=\sum_{j=1}^{N_{S P}} S_{j, v} h_{j}\left(T_{s}\right)
$$


where the total molar flow of species $j$ carried in the volatiles, $S_{j, v}$, is given by Eq. (17). We make the assumption that this volatiles gases are released at the temperature $T_{s}$ of the solid phase. Note that the solid phase is characterized by a common local temperature as discussed in subsection 2.4.

The heterogeneous reactions involve the adsorption of gaseous species into the fuel particles, and the desorption of the reaction products back to the gaseous phase. Using the heterogeneous reaction model described in sub-section 2.1.3, we have that for the $\gamma$-th of such reactions, the enthalpy transport associated with those adsorbed and desorbed flows could be in principle estimated as

$$
\frac{r_{\gamma}^{(H)}}{W_{\mathrm{S}}} \sum_{j=1}^{N_{S P}} v_{j \gamma}^{\prime} h_{j}\left(T_{g}\right) \text { and } \frac{r_{\gamma}^{(H)}}{W_{\mathrm{S}}} \sum_{j=1}^{N_{S P}} v_{j \gamma}^{\prime \prime} h_{j}\left(T^{\prime \prime}\right), \text { respectively. }
$$

In the first expression the enthalpies of the incoming species can be evaluated at the temperature $T_{g}$ of the gas in the neighborhood of the fuel particle. In the second expression however the enthalpies of the outgoing products should be evaluated at a temperature $T^{\prime \prime}$ that would have to be approximated in some way. Such an estimation is a difficult task, so that a practical approach is to consider that a fraction $\varepsilon$ of the heat reaction is retained in the solid particle, contributing to its internal energy, while the remaining fraction $(1-\varepsilon)$ goes into the gas phase as the net flow of sensible enthalpy. More explicitly, the heat released by the $\gamma$ th heterogeneous reaction in the solid is

$$
\begin{aligned}
Q_{s \gamma} & =\frac{r_{\gamma}^{(H)}}{W_{S}}\left\{\sum_{j=1}^{N_{S P}}\left(v_{j \gamma}^{\prime} h_{j}\left(T_{g}\right)-v_{j \gamma}^{\prime \prime} h_{j}\left(T^{\prime \prime}\right)\right)+h_{f S}^{o}\right\} \\
& =\frac{r_{\gamma}^{(H)}}{W_{S}} \underbrace{\sum_{j=1}^{N_{S P}}\left(v_{j \gamma}^{\prime \prime}-v_{j \gamma}^{\prime}\right) h_{f j}^{o}-h_{f S}^{o}}_{Q_{R \gamma}}+\underbrace{r_{\gamma}^{(H)}\left(\sum_{S=1}^{N_{S P}} v_{j \gamma}^{\prime} \Delta h_{j}\left(T_{g}\right)-\sum_{j=1}^{N_{S P}} v_{j \gamma}^{\prime \prime} \Delta h_{j}\left(T^{\prime \prime}\right)\right)}_{H_{V, H E \gamma}}
\end{aligned}
$$

with $Q_{R_{\gamma}}$ being the standard heat of reaction and $\Delta h_{j}$ are sensible enthalpies. On the other hand, we assume that

$$
Q_{s \gamma}=-\varepsilon \frac{r_{\gamma}^{(H)}}{W_{\mathrm{S}}} Q_{R \gamma}
$$

(By definition, $Q_{R y}$ is negative for exothermic reactions, whence the minus sign in Eq. (39)). As a result, the net flow of energy toward the gas phase is

$$
H_{V, H E}=\sum_{\gamma=1}^{N_{H E}} H_{V, H E \gamma}=-(1-\varepsilon) \frac{1}{W_{\mathrm{S}}} \sum_{\gamma=1}^{N_{H E}} r_{\gamma}^{(H)} Q_{R \gamma}
$$

We look now to the third component of $H_{V}$, namely the convective heat transfer to the gas on the surface of the solid medium. This heat flow can be calculated, in a simplified form, as 


$$
H_{V, Q}=\xi F\left(T_{s}-T_{g}\right)
$$

where $\zeta$ is the heat transfer coefficient, which can be estimated from empirical relations for porous media, as for example [33]

$$
\mathrm{Nu}=2+1.1 \operatorname{Re}^{0.6} \operatorname{Pr}_{g}^{1 / 3}
$$

In this expression the characteristic length for the Reynolds and Nusselt numbers is taken as two times the particles size $d$, and the velocity is the effective mean velocity of the flow:

$$
\operatorname{Re}=\frac{\theta|\overrightarrow{\mathbf{u}}| 2 d}{v_{g}}, \quad \mathrm{Nu}=\frac{\xi 2 d}{\lambda_{g}}
$$

Upon substituting Eq. (36) into (35), the equation for energy conservation in the gas phase becomes

$$
\frac{\partial}{\partial t}\left(\theta \rho_{g} h_{g}\right)+\nabla \cdot\left(\rho_{g} h_{g} \overrightarrow{\mathbf{u}}\right)=\nabla \cdot\left(\theta \lambda_{g} \nabla T_{g}\right)+\nabla \cdot\left(\theta \rho_{g} \sum_{j=1}^{N_{S P}} h_{j} D_{j} \nabla y_{j}\right)+H_{V, v}+H_{V, H E}+H_{V, Q}
$$

Recall that we are using total enthalpies $h$, which include the enthalpy of formation, so that the heat released by the homogeneous reactions in the gaseous phase is implicit in Eq. (44).

This equation of energy, coupled with the equations for mass conservation of species, and the equations for the solid phase, give rise to a system of simultaneous partial differential equations that must be solved by a numerical iterative scheme. In that case Eq. (44) can be solved for $h_{g}$ as its primitive variable, and the gas temperature can be obtained by solving the polynomial equation

$$
\sum_{n=0}^{m} c_{n j} T_{g}^{n}=h_{g} \text { with } c_{n j}=\sum_{j=1}^{N_{S P}} a_{n j} y_{j}
$$

in each iteration. This equation for $T_{g}$ results from Eqs. (32) and (12).

\subsection{Conservation of energy of the solid phase}

The conservation of energy for the solid phase can be analyzed in the same volume $V(t)$ used in subsection 2.3, with a boundary that moves at the velocity ( $\overrightarrow{\mathbf{v}}$ ) of propagation of solid fuel consumption. In this way we have

$$
\frac{\mathrm{d}}{\mathrm{d} t} \int_{V(t)}\left(\rho_{f} e_{f}+\rho_{i} e_{i}\right)(1-\theta) \mathrm{d} V=\int_{V(t)} G_{s} \mathrm{~d} V+\int_{A(t)} H_{s} \mathrm{~d} A_{s}
$$

or equivalently 


$$
\int_{V(t)} \frac{\partial}{\partial t}\left((1-\theta)\left(\rho_{f} e_{f}+\rho_{i} e_{i}\right)\right) \mathrm{d} V+\int_{A(t)}(1-\theta)\left(\rho_{f} e_{f}+\rho_{i} e_{i}\right) \overrightarrow{\mathbf{v}} \cdot \overrightarrow{\mathbf{n}} \mathrm{d} A=\int_{V(t)} G_{s} \mathrm{~d} V+\int_{A(t)} H_{s} \mathrm{~d} A_{s}
$$

where $e_{f}, e_{i}$ are the internal energies of fuel and inert particles respectively, $G_{s}$ is the total source of energy for the solid medium per unit of bulk volume, and $H_{s}$ is the influx of energy on the boundary surface per unit of solid area. Because of the thorough mix and contact between inert and fuel particles, we assume that they are in local thermal equilibrium. Consequently, the thermal state of the solid medium can be characterized by only one local temperature $T_{s}$. If that is not the case (for instance, if the specific heats of the inert and fuel particles were too different), separate energy equations for both the fuel and inert material can be formulated.

The source $G_{s}$ is determined by the convective heat transfer between the solid and the gas, and the fraction of the heat released by the heterogeneous reactions that has remained in the solid phase. According to Eqs. (39) and (41) we have

$$
G_{s}=-\frac{\varepsilon}{W_{S}} \sum_{\gamma=1}^{N_{H E}} r_{\gamma}^{(H)} Q_{R \gamma}-\xi F\left(T_{s}-T_{g}\right)
$$

The influx $H_{s}$ is given essentially by heat conduction through the solid medium. This can be written as $H_{s}=-\overrightarrow{\mathbf{q}}_{s} \cdot \overrightarrow{\mathbf{n}}$, with the heat flux $\overrightarrow{\mathbf{q}}_{s}=-\lambda_{s} \nabla T_{s}$, and $\lambda_{s}$ being the equivalent thermal conductivity of the solid medium.

Upon substituting these expression into Eq. (47), and converting the surface integral into volume integral, the equation for energy conservation of the solid phase becomes

$$
\begin{aligned}
& \frac{\partial}{\partial t}\left((1-\theta)\left(\rho_{f} C_{f}+\rho_{i} C_{i}\right) T_{s}\right)+\nabla \cdot\left((1-\theta)\left(\rho_{f} C_{f}+\rho_{i} C_{i}\right) \overrightarrow{\mathbf{v}} T_{s}\right)=\nabla \cdot\left((1-\theta) \lambda_{s} \nabla T_{s}\right) \\
&-\frac{\varepsilon}{W_{S}} \sum_{\gamma=1}^{N_{H E}} r_{\gamma}^{(H)} Q_{R \gamma}-\xi F\left(T_{s}-T_{g}\right)
\end{aligned}
$$

\subsection{Summary}

In this section, we have presented the formulation of a mathematical model for hybrid filtration combustion, along with some model assumptions that would allow a computational approach. Such treatment will depend of course on the boundary and initial conditions for particular reactor designs. As we have shown, a crucial factor for the modelling of these complex phenomena is the availability of model parameters such as $\varepsilon$, the composition of the volatiles $V_{\beta}$, etc. The parameterization of a model of this nature requires extensive experimental work. Our purpose is not to derive here all these parameters, but to show some experimental results that highlight the potential of ultra-rich hybrid filtration combustion for hydrogen and syngas production. 


\section{Hydrogen production by hybrid filtration combustion}

In this section, we show experimental results for wave velocities, combustion temperature, and $\mathrm{H}_{2}$ and $\mathrm{CO}$ concentrations for hybrid combustion of gas and solid fuels in porous media.

\subsection{Natural gas and coal particles}

The porous medium is composed of uniformly mixed aleatory coal and alumina spheres with varying volume fractions. This section provide the data on natural gas-air flames stabilized inside hybrid porous media with a volumetric coal content from 0 to $75 \%$ for an equivalence ratio $\phi=2.3$ and a filtration velocity of $15 \mathrm{~cm} / \mathrm{s}$.

The solid temperatures recorded for hybrid filtration combustion in coal-alumina beds, decreased from $1537 \mathrm{~K}$ at $15 \%$ of coal in porous media to $1217 \mathrm{~K}$ at $75 \%$ (Fig. 1a). The wave velocity is reduced with increase of coal volume fraction. At high coal contents the wave velocity is limited by the oxygen availability and the bed displacement resulting from the coal consumption. The latter factor suggests that the wave velocity in the packed bed with $100 \%$ coal content will be close to zero.

The hydrogen concentration increased with an increase of coal content with the maximum of $22 \%$ at a coal content of $75 \%$. The result shows that high combustion temperatures facilitate hydrogen production in hybrid filtration combustion. The carbon monoxide also increased with an increase of coal content.

The maximum hydrogen conversion reached $55 \%$ at a coal content of $75 \%$ compare to $35.1 \%$ for natural gas flames propagating in the inert porous bed (Fig. 1b).

\subsection{Butane and wood pellets}

The porous media was composed of uniformly mixed aleatory wood pellets and alumina spheres. The equal volumes of $5.6 \mathrm{~mm}$ solid alumina balls and wood pellets were mixed resulting in the packet bed with a porosity of $\sim 40 \%$. Experimental data were collected for a slightly increasing filtration velocity in a range of equivalence ratios from stoichiometry $(\phi=$ 1.0) to $\phi=2.6$.

The solid temperature of butane/air mixtures in the inert porous medium increases from $1510 \mathrm{~K}$ at $\phi=1.45$ to $1610 \mathrm{~K}$ at $\phi=2.6$ (Fig. 2a). The solid temperatures recorded for hybrid filtration combustion in wood pellets-alumina spheres porous media, decrease from 1477 $\mathrm{K}$ at $\phi=1.45$ to $1216 \mathrm{~K}$ at $\phi=2.6$. For butane/air mixtures in the inert and composite porous media the maximum absolute velocity value of $\sim 0.012 \mathrm{~cm} / \mathrm{s}$ is observed for $\phi=1.45$ (Fig. 2b).

Hydrogen concentration increases with an increase of equivalence ratio for butane/air mixtures in the inert porous medium. In comparison, more than four times higher concentration of hydrogen is measured for butane/air mixtures in the composite porous 
media made of alumina and wood pellets. For butane/air mixtures in the inert porous medium the concentration of carbon monoxide increased with equivalence ratio and for aninert porous media the concentration of $\mathrm{CO}$ remains almost constant with the equivalence ratio.

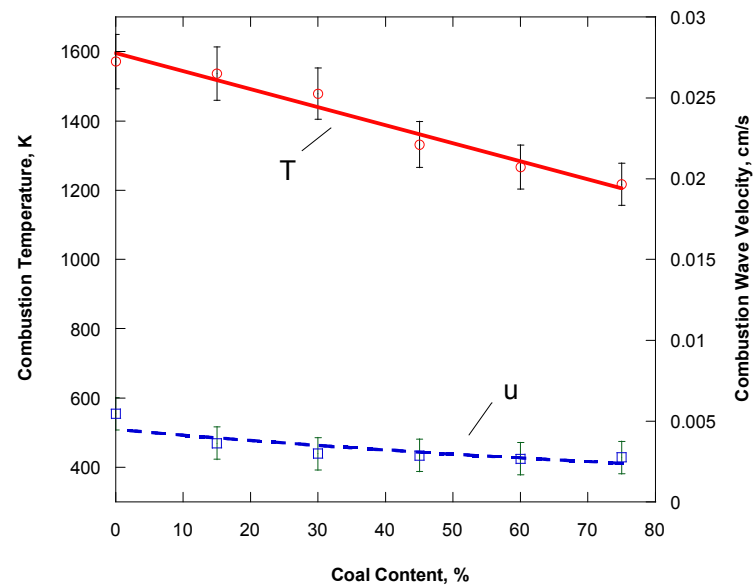

A

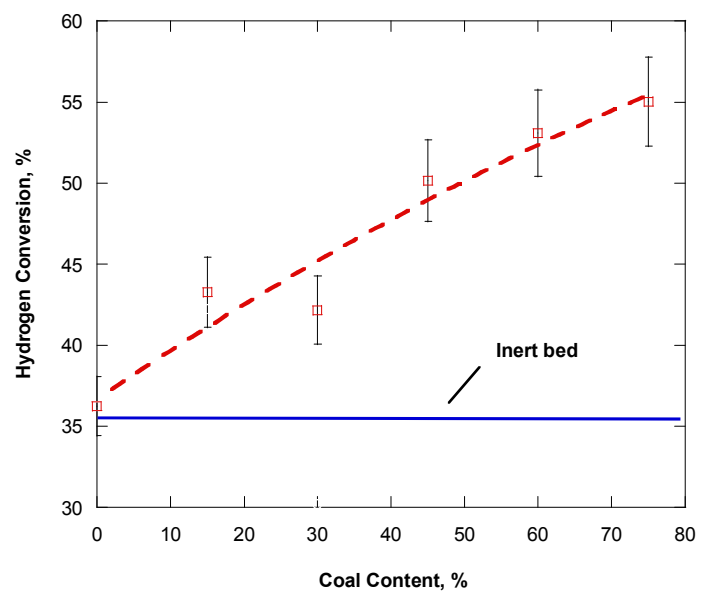

B

Figure 1. Combustion temperatures and wave velocities (A) and degree of conversion to hydrogen (B) for rich and ultra-rich waves for natural gas mixtures with air varying the volume of coal in the porous media from 0 to $75 \%$ [34].

The hydrogen yield is calculated using the initial hydrogen content in butane for the case of inert bed and the initial hydrogen content in butane and wood pellets for the case of the composite bed. The maximum yield recorded for the inert porous medium is close to $10 \%$ at $\phi=2.6$ (Fig. 2c). The maximum yield recorded for the composite pellets is $\sim 48 \%$ at $\phi=2.4$. 

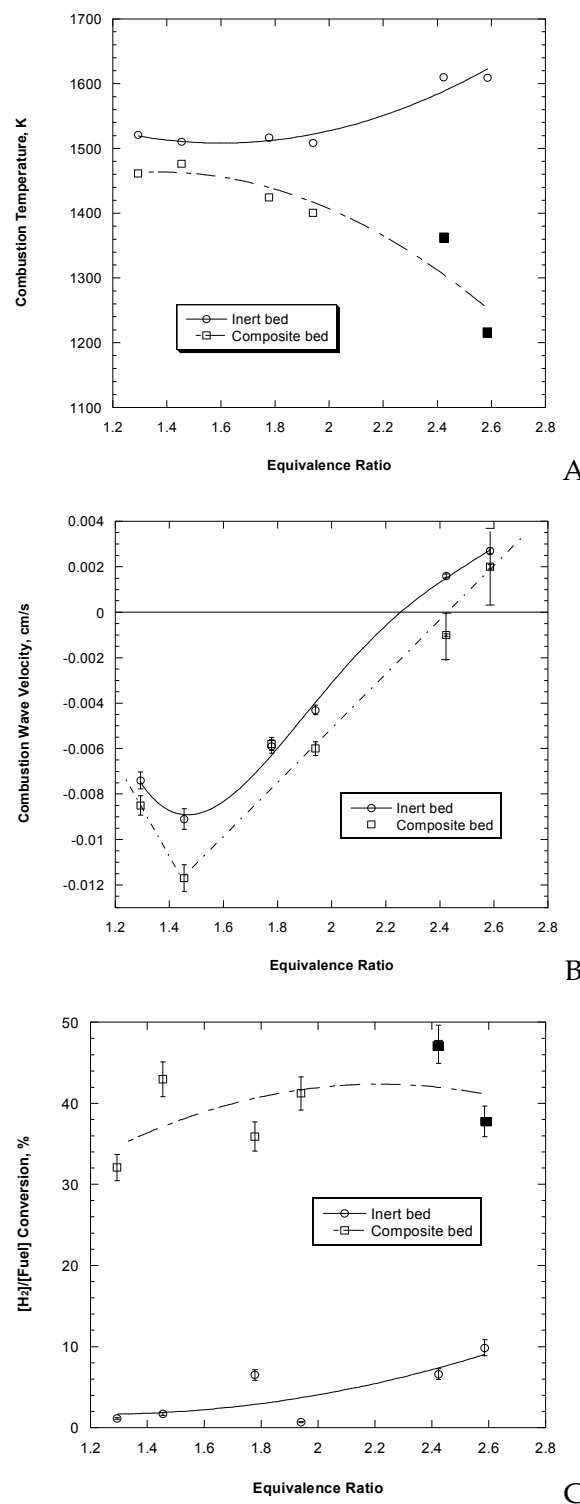

Figure 2. Combustion temperatures (A), wave velocities (B) and degree of conversion to hydrogen (C) for rich and ultra-rich waves for butane mixtures with air in the inert bed and composite porous media [35].

\subsection{Propane and polyethylene pellets}

The reactor is filled with a uniformly mixed aleatory polyethylene pellets and alumina spheres whose diameter is $5.6 \mathrm{~mm}$. The geometry of polyethylene pellets are $2.4 \times 5.0 \times 4.4$ 
$\mathrm{mm}$. Experimental data were collected at a range of equivalence ratios $(\phi)$ from $\phi=1.0$ to $\phi$ $=1.7$. The air/propane flow rate was maintained constant at $5.7 \mathrm{~L} / \mathrm{min}$ yielding a filtration velocity of $11.3 \mathrm{~cm} / \mathrm{s}$.
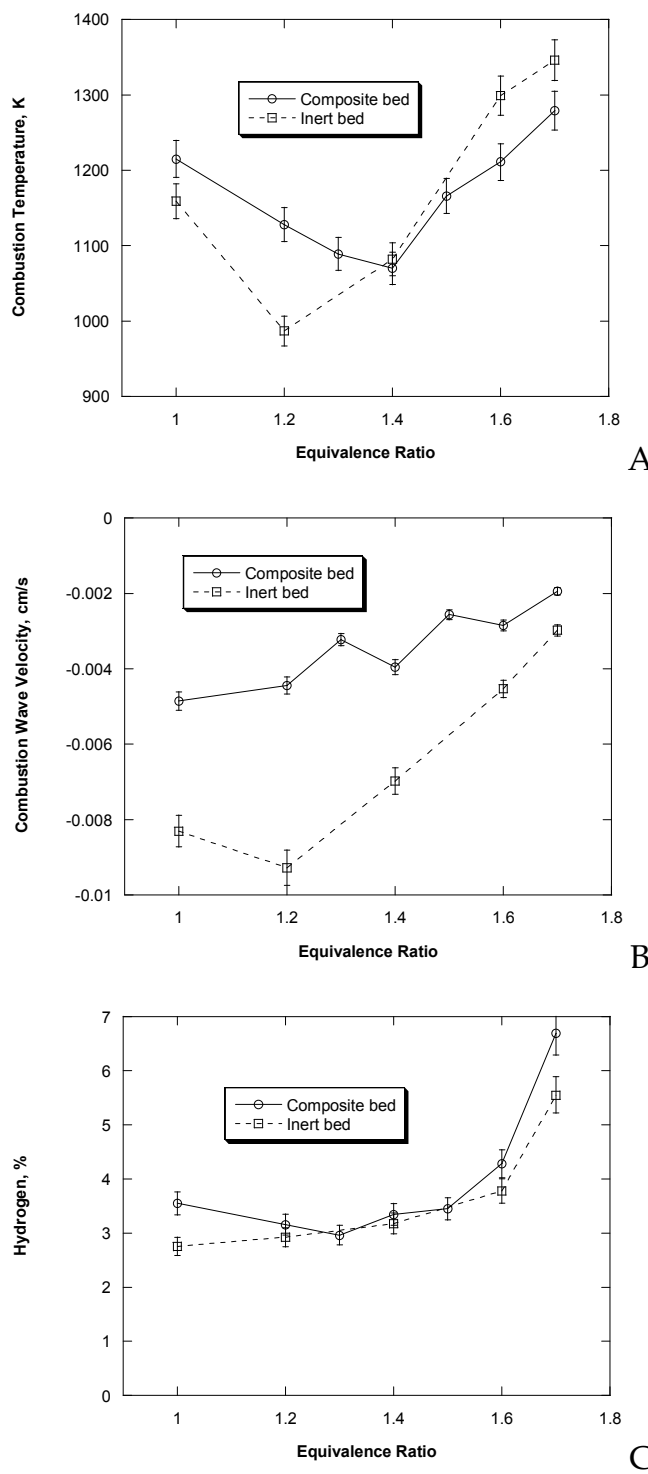

Figure 3. Combustion temperatures (A), wave velocities (B) and hydrogen concentration (C) in rich mixtures of propane with air for gaseous and hybrid filtration combustion waves. 
The solid temperature of propane/air mixtures in the inert porous medium decreases from $1159 \mathrm{~K}$ at $\phi=1.0$ to $987 \mathrm{~K}$ at $\phi=1.2$ (Fig. 3a). Then the temperature increase to $1346 \mathrm{~K}$ at $\phi=$ 1.7. The solid temperatures recorded for hybrid filtration combustion in polyethylene pellets-alumina spheres porous media, shows similar behaviour and decrease from $1215 \mathrm{~K}$ at $\phi=1.0$ to $1070 \mathrm{~K}$ at $\phi=1.4$. Then the temperature increase to $1279 \mathrm{~K}$ at $\phi=1.7$. The experimental results for propane/air mixtures in the inert and composite porous media upstream wave were recorded in all the range of equivalence ratios studied (Fig. 3b).

Hydrogen concentration increases with an increase of equivalence ratio for propane/air mixtures in the inert porous medium and composite porous media made of alumina and polyethylene pellets. The maximum generated mole fraction of hydrogen is $6.7 \%$ for hybrid propane. Carbon monoxide is also generated in both porous media reactor.

\section{Conclusion}

In this chapter we have defined the hybrid filtration combustion process. The physical phenomena involved as well as their mathematical description by the governing equations enforcing mass and energy conservation for both the gas and solid phases are discussed.

We presented experimental evidence that such a process of hybrid filtration combustion can actually be achieved in practice. In particular we showed applications for reformation of gaseous and solid fuels into hydrogen and syngas. This was shown for several combinations of gaseous and solid fuels.

\section{Author details}

Mario Toledo Torres* and Carlos Rosales Huerta

Department of Mechanical Engineering, Technical University Federico Santa Maria, Valparaiso, Chile

\section{Acknowledgement}

The authors wish to acknowledge the support by the CONICYT-Chile (FONDECYT 1121188 and BASAL FB0821 - FB/05MT/11).

\section{References}

[1] Kaviany M (1995) Principles of heat transfer in a porous media. Springer-Verlag. New York.

[2] Toledo M, Bubnovich V, Saveliev A, Kennedy L (2009) Hydrogen production in ultrarich combustion of hydrocarbon fuels in porous media. Int. J. Hydrogen Energy 34: 1818-1827.

\footnotetext{
${ }^{*}$ Corresponding Author
} 
[3] Dhamrat RS, Ellzey JL (2006) Numerical and experimental study of the conversion of methane to hydrogen in a porous media reactor. Combust. Flame 144: 698-709.

[4] Babkin V, Korzhavin A, Bunev V (1991) Propagation of premixed gaseous explosion flames in porous media. Combust. Flame 87: 182-190.

[5] Babkin V. (1993) Filtrational combustion of gases. Present state of affairs and prospects. Pure Appl. Chem. 65: 335-344.

[6] Vogel BJ, Ellzey JL (2005) Subadiabatic and superadiabatic performance of a two-section porous burner. Combust. Sci. Technol. 177: 1323-1338.

[7] Abdul M, Abdullaha M, Abu Bakarb M (2009) Combustion in porous media and its applications - a comprehensive survey. J. Environ. Manage 90: 2287-2312.

[8] Bingue JP, Saveliev AV, Fridman AA, Kennedy LA (2002) Hydrogen production in ultra-rich filtration combustion of methane and hydrogen sulfide. Int. J. Hydrogen Energy 27: 643-649.

[9] Babkin V (1993) Filtrational combustion of gases. Pure and Applied Chemistry 65: 335344.

[10] Dobrego KV, Zhdanok SA, Khanevich EI (2000) Analytical and experimental investigation of the transition from low velocity to high-velocity regime of filtration combustion. Exp. Therm. Fluid. Sci. 21: 9-16.

[11] Foutko SI, Shabunya SI, Zhdanok SA, Kennedy LA (1996) Superadiabatic combustion wave in a diluted methane-air mixture under filtration in a packed bed. Proc. Combust. Inst. 25: 1556-65.

[12] Kennedy LA, Bingue JP, Saveliev AV, Fridman AA, Foutko SI (2000) Chemical structures of methane-air filtration combustion waves for fuel-lean and fuel-rich conditions. Proc. Combust. Inst. 28: 1431-1438.

[13] Drayton MK, Saveliev AV, Kennedy LA, Fridman AA, Li Y- E (1998) Syngas production using superadiabatic combustiom of ultra-rich methane-air mixtures. Proc Combust. Inst. 27: 1361-7.

[14] Weinberg FJ, Bartleet TG, Carleton FB, Rimbotti P, Brophy JH, Manning RP (1988) Partial oxidation of fuel rich mixtures in a spouted bed combustor. Combust Flame 72: 235-9.

[15] Ytaya Y, Oyashiki T, Hasatani M (2002) Hydrogen production by methane-rich combustion in a ceramic burner. J. Chem. Eng. Jpn. 35: 46-56.

[16] Kennedy LA, Saveliev AV, Fridman AA (1999) Transient filtration combustion. Mediterr. Combust. Symp. 1: 105-39.

[17] Kennedy LA, Saveliev AA, Bingue JP, Fridman AA (2002) Filtration combustion of a methane wave in air for oxygen enriched and oxygen-depleted environments. Proc. Combust. Inst. 29: 835-41.

[18] Howell JR, Hall MJ, Ellzey JL (1996) Combustion of hydrocarbon fuels within porous medium. Prog. Energy Combust. Sci. 22: 121-45. 
[19] Gavrilyuk VV, Dmitrienko YM, Zhdanok SA, Minkina VG, Shabunya SI, Yadrevskaya NL, et al. (2001) Conversion of methane to hydrogen under superadiabatic filtration combustion. Theor. Found. Chem. Eng. 35: 589-96.

[20] Gerasev AP (2008) Hybrid autowaves in filtration combustion of gases in a catalytic fixed bed. Combust. Explos. 44: 123-32.

[21] Schoegl I, Newcomb SR, Ellzey JL (2009) Ultra-rich combustion in parallel channels to produce hydrogen-rich syngas from propane. Int. J. Hydrogen Energy 34: 5152-63.

[22] Dixon MJ, Schoegl I, Hull CB, Ellzey JL (2008) Experimental and numerical conversion of liquid heptane to syngas through combustion in porous media. Combust. Flame 154: 217-31.

[23] Pedersen-Mjaanes H, Chan L, Mastorakos E (2005) Hydrogen production from rich combustion in porous media. Int. J. Hydrogen Energy 30: 579-92.

[24] Tarun K. Kayal, Mithiles Chakravarty (2007) Modeling of a conceptual self-sustained liquid fuel vaporization-combustion system with radiative output using inert porous media, International Journal of Heat and Mass Transfer 50: 1715-1722.

[25] Sumrerng Jugjai, Nopporn Polmart (2003) Enhancement of evaporation and combustion of liquid fuels through porous media, Experimental Thermal and Fluid Science 27: 901909.

[26] Trimis D, Wawrzinek K, Hatzfeld O, Lucka K, Rutsche A, Haase F, Kruger K, and Kuchen C (2001) High modulation burner for liquid fuels based on porous media combustion and cool flame vaporization. Proceedings of the Sixth International Conference on Technologies and combustion for clean environment, v.2, Porto, Portugal, 9-12 July (Ed. M. G. Carvhalho), pp. 1-8.

[27] Tarun K. Kayal, Mithiles Chakravarty (2005) Combustion of liquid fuel inside inert porous media: an analytical approach. International Journal of Heat and Mass Transfer $48: 331-339$.

[28] Haack DP (1993) Mathematical analysis of radiatively enhanced liquid droplet vaporization and liquid fuel combustion within a porous inert medium. MSc Thesis, University of Texas, Austin.

[29] Kaplan M and Hall MJ (1995) The combustion of liquid fuels within a porous media radiant burner. Exp. Thermal. Fluid Sci. 11: 13-20.

[30] Tseng CJ and Howell JR (1994) Liquid fuel combustion within inert porous media. Heat Trans. Combined Modes, ASME, HTD 299 : 63-69.

[31] Salganskii EA, Fursov VP, Glazov SV, Salganskaya MV, Manelis GB (2006) Model of vaporeair gasification of a solid fuel in a filtration mode. Combust. Explos. 42: 55-62.

[32] Poinsot T, and Veynante D (2001) Theoretical and Numerical Combustion. R. T. Edwards Inc. Philadelphia.

[33] Salgansky EA, Kislov VM, Glazov SV, Zholudev AF, Manelis GB (2008) Filtration Combustion of a Carbon-Inert Material System in the Regime with Superadiabatic Heating. Combustion, Explosion, and Shock Waves 44: 273-280. 
[34] Toledo MG, Utria KS, González FA, Zúñiga JP, Saveliev AV (2012) Hybrid filtration combustion of natural gas and coal. Int. J. Hydrogen Energy 37 : 6942-6948.

[35] Toledo M, Vergara E, Saveliev A (2011) Syngas production in hybrid filtration combustion. Int. J. Hydrogen Energy 36: 3907-3912. 